\title{
Structure matters
}

Energy Environ. Sci. https://doi.org/10.1039/C9EE01872G (2019)

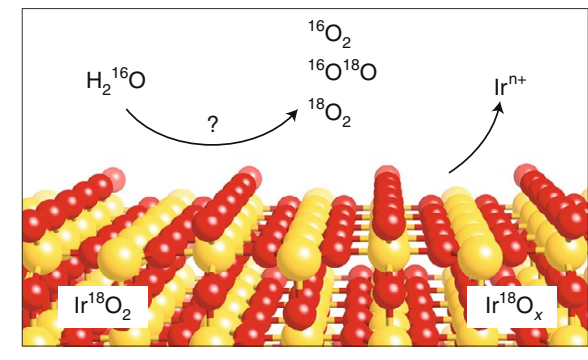

The future success of clean fuels such as hydrogen will, in part, depend on their efficient and economically viable large-scale generation. Water electrolysers are meant to play a big role, and, in particular, acidic proton-exchange membrane electrolysers hold great promise due to their high activity and efficiency. However, currently less than $5 \%$ of industrially produced hydrogen comes from water electrolysis. Protonexchange membrane electrolysers rely on costly iridium-based anode catalysts; understanding the stability and degradation mechanisms of such catalysts is therefore vital to ensure their successful optimization.

Now, Karl Mayrhofer, Baptiste Gault, Olga Kasian and colleagues use atom probe tomography and on-line electrochemical mass spectrometry on two iridium-oxide catalysts to explore their catalytic and degradation mechanisms during acidic water oxidation. The catalysts investigated are reactively sputtered, rutile $\mathrm{IrO}_{2}$ and amorphous, hydrous $\mathrm{IrO}_{x} \mathrm{H}_{y}$. Atom probe tomography shows that the hydrous oxide contains a network of nanopores with $\mathrm{Ir}^{\mathrm{III}} \mathrm{OOH}$ groups on near-surface regions, whereas the rutile oxide has a compact structure with no $\mathrm{OH}$ groups. The two catalysts are then isotopically labelled with ${ }^{18} \mathrm{O}$ and the evolution of ${ }^{16} \mathrm{O}_{2},{ }^{16} \mathrm{O}^{18} \mathrm{O}$ and ${ }^{18} \mathrm{O}_{2}$, as well as Ir dissolution, are monitored by on-line electrochemical mass spectrometry in $0.1 \mathrm{M} \mathrm{HClO}_{4}$ in $\mathrm{H}_{2}{ }^{16} \mathrm{O}$ during anodic scans or galvanostatic anodic polarization.

The results indicate participation of lattice oxygen during the oxygen evolution reaction on the hydrous oxide, which contributes as ${ }^{16} \mathrm{O}^{18} \mathrm{O}$ to $0.5 \%$ of total current density. ${ }^{18} \mathrm{O}_{2}$ evolution and Ir dissolution are appreciable, but their estimated partial current densities are one and two orders of magnitude lower, respectively, and furthermore, their ratio is always constant, which suggests that both processes are connected. On the other hand, evolution of lattice oxygen and Ir dissolution are insignificant on the rutile oxide. The researchers attribute the lattice oxygen participation and linked degradation to the structural differences between both oxides, in particular the porosity and presence of defects and $\mathrm{Ir}^{\mathrm{III}} \mathrm{OOH}$ groups.

\section{Marçal Capdevila-Cortada}

Published online: 14 November 2019 https://doi.org/10.1038/s41929-019-0390-8 